\title{
MATHEMATICAL MODELING OF FINDING EFFECTIVE ALGORITHMS OF TRANSPORT COMPANY FUNCTIONING
}

\author{
Andrey Levterov \\ Ph.D., Professor, Kharkiv National Avtomobile \& Highway University, Ukraine \\ e-mail: lai@khadi.kharkov.ua, orcid.org/0000-0001-6586-1061 \\ Larisa Kozachok \\ Senior Lecture, Kharkiv National Avtomobile \& Highway University, Ukraine \\ e-mail: LarisaK2010@ukr.net, orcid.org/0000-0002-5246-4240 \\ Marina Kostikova \\ Ph.D., Kharkiv National Avtomobile \& Highway University, Ukraine \\ e-mail: kmv_topaz@ukr.net,orcid.org/0000-0001-5197-7389
}

\section{Summary}

This article is devoted to the study of certain stages of transport enterprises operation processes. The subject of consideration is the technological processes of production of a certain type of products at a single transport enterprise during the technological process in the mechanical assembly shop. To fully represent and consideration of all factors of influence, the enterprise was considered as a human-machine system and further, during the research, an analysis of the structure of the system and the principles of its operation was carried out. This article provides a model of a certain technological process, which, despite its simplicity, is so meaningful that it encompasses a wide range of practical optimization tasks.

Keywords: technological processes, system structure, mathematical model, transport company, algorithmization, operating schedule.

DOI: https://doi.org/10.23856/4536

\section{Introduction}

When considering practical tasks or studying processes, systems with different service routes for requirements are most widespread. As noted by V. Dudek, M. Smith and S. Panwalkar, in $60 \%$ of the surveyed industrial companies, demand maintenance routes were different (Dudek R.A., Smith M.L., Panwalkar S.S., 1974).

V. G. Timkovsky, N. Hifitz and I. Adiri proposed polynomial algorithms for constructing an optimal service schedule with two devices n simultaneously incoming requirements with single duration of operations (Timkovsky, V.G., Hifitz N. and Adiri I., 1982). The labor intensity of these algorithms is $O(R)$, where $R$ is the total number of operations performed.

A fairly complete account of the theory of duality in relation to the problems of constructing optimal schedules is contained in the monograph by V.S. Mikhalevich and A.I. Kuksa (Mikhalevich V.S., Kuksa A.I., 1983). J. Peter van Dam, Gerard J. C. Gaalman noted, investigated and justified the planning and execution of technological processes in enterprises, based on schedule theory, which relates to the study of operations. In the work, it was noted that these planning and management tasks are combinatorily complex due to many factors: performing technological operations on the equipment of in-line workshops with limitations in the time of intermediate storage, the presence of manual operations, and 
common resources for all technological processes. The planning algorithm that is developed is based on the characteristics of multilevel scheduling taking into account the hierarchy of levels (J. Peter van Dam, Gerard J. C. Gaalman, 1994). The best known methods of sequential analysis of variants was developed by M.L. Fisher, B.J. Lageweg, J.K. Lenstra - this is a method of branches and bounds (Fisher M.L., Lageweg B.J., Lenstra J.K., 1983).

\section{Purpose and methods of research}

The article focuses on the mathematical setting of the problem and the construction of a mathematical model of a technological process at the vehicle transport enterprise. In order to find the optimal structure and select the most effective options for performing technological processes and managing them, break the system into subsystems and processes.

When examining the production technologies of a particular system, we consider the issues of ordering the service of successive technological processes according to the criterion of minimizing the time spent in the system, as well as the order of service associated with the execution of processes with restrictions in the form of policy or deadlines.

\section{Mathematical model and problem setting}

The mathematical model of the technological problem is compiled in order to find at the given properties of tasks and resources and the restrictions imposed on them an effective algorithm for ordering tasks that optimizes or seeks to optimize the desired efficiency measure. The measure of efficiency in this task will be understood as the length of the schedule and the average time of the tasks in the system (the meaning of these concepts will be explained below).

To build a model, let's take a look at the main steps in implementing the process, which is provided by a set of processors $P=\left\{P_{1}, P_{2}, \ldots, P_{m}\right\}$, which differ in our case in terms of capabilities, performance and functional operations. A common job system $T=\left\{T_{1}, T_{2}, \ldots, T_{n}\right\}$ for a given set of processors is a set of jobs to be executed, on which a partial order relationship can be determined: the $T_{i}<T_{j}$ entry means that the $T_{i}$ job must be completed earlier in time than the $T_{j}$ job starts.

To fully describe the jobs maintenance process, we will introduce the concept of a system functioning schedule, a set of instructions for determining which jobs are serviced, serviced or will be serviced at each point in time.

From the theory of schedules, the following types of schedules are known:

1. No-interrupt schedules that assume that every job that starts cannot be interrupted, that is, the job must always be completed.

2. Interrupt schedules allow jobs to be aborted and delayed by the processor, and it is assumed that the total time required to complete the job remains unchanged and there is no loss of service time during interrupts.

3. Schedules using the list assume that an ordered list of jobs from the set of $T$, the so-called priority list, is first prepared, when the processor is released, the list is viewed first until there is the first failed job that is ready to run. A job is considered ready to run on a given processor if all preceding jobs have completed and the available resources are sufficient to run.

A valid schedule is a schedule that satisfies all the limitations arising from setting a specific task under consideration.

To take advantage of the schedule efficiency measure, introduce the concept of schedule length. 
The length of the schedule for the set (1) will be the time required to complete the entire set of jobs

$$
T=\left\{T_{1}, T_{2}, \ldots, T_{n}\right\}, T_{i}<T_{j}, i<j ; i=\overline{1, n} ; j=\overline{1, n}
$$

The length of the schedule depends on the total execution time of all jobs and the total idle time of the processors. The lowest-length schedule corresponds to a situation where there are no processors downtimes.

In the future, it is also necessary to use the concept of work $R_{j}$, which is an ordered sequence of jobs $T_{j}, j=\overline{1, k}$ and belongs to the set (2):

$$
W=\left\{R_{1}, R_{2}, \ldots, R_{j}, \ldots, R_{k}\right\}
$$

The schedule can be considered as a set $S=\left\{S_{1}(t), S_{2}(t), \ldots, S_{l}(t), \ldots, S_{m}(t)\right\}$ of piecemeal-constant continuous functions on the left $S_{l}(t), l=\overline{1, m}$, each of which takes values $0,1,2, \ldots, n$.

Consider, that $S_{l}\left(t^{\prime}\right)=i \neq 0$, if at time $t=t^{\prime}$ the processor $P_{l}$ serves the job $T_{i}$; and $S_{l}\left(t^{\prime}\right)=0$, if at time $t=t^{\prime}$ the processor $P_{l}$ is idle.

The processor system serving the job data - the serving system - is called one-step if each job can be fully serviced by one of the processors. The service times $t_{i l}$ of each job $T_{i}, i=\overline{1, n}$ by each processor $P_{l}, l=\overline{1, m}$ are assumed to be set.

The service system is called multistage if the maintenance process of the $T_{i}$ job includes $r_{i}$ consecutive stages, with each stage corresponding to a set of $M_{r_{i}}$, whose members are processors from the set $P=\left\{P_{1}, P_{2}, \ldots, P_{m}\right\}$.

If $\left|M_{r_{i}}\right|=1$, the service system is called a serial processor system. In this case, for each $T_{i}$ job, the path of the processors $L^{i}=\left\{P_{1}, P_{2}, \ldots, P_{r_{i}}\right\}$ is set. Among systems with serial processors, stream-type systems are distinguished. In these systems, each job must be serviced sequentially by each processor, the processor paths are the same for all jobs. In systems with non-fixed routes, the order of processor passage is not predetermined, each jobs "selects" it at its own discretion.

In any case, if the job $T_{i}$ in the stage $r_{i}$ is served by the processor $P_{l}, l=\overline{1, m}$, then the predetermined duration of its service time $t_{i l}$ is assumed.

Elements of multiple processors of one stage are called parallel. Concurrently, the non-interruptive job maintenance process on a parallel processor system satisfies the following condition:

- each job is serviced by only one processor. If the maintenance of the $T_{i}$ job by the processor $P_{l}$ starts at time $t_{i}^{0}$ it proceeds continuously and ends at time $t_{i}=t_{i}^{0}+t_{i l}$.

\section{Research of the functioning processes}

We set the task of describing the technological process of manufacturing a certain assembly of parts included in the production scheme of vehicle transport enterprise. When considering routings, we will limit ourselves to three types of parts, which are then assembled into a specific technical unit. Based on this, a set of jobs of the maintenance system, the manufacture of this technical part, can be presented in the form of three subsets.

Let the definitions of the first subset

$T^{1}=\left\{T_{1}{ }_{1}, T^{\prime}{ }_{2}, \ldots, T^{\prime}{ }_{l}\right\}-$ correspond to the parts of the first type details;

$T^{2}=\left\{T_{1}, T_{2}, \ldots, T_{l}\right\}-$ parts of the second type; 
$T^{3}=\left\{T^{\prime \prime \prime}, T^{\prime \prime \prime}{ }_{2}, \ldots, T^{\prime \prime \prime}{ }_{l}\right\}-$ parts of the third type.

where $l-$ is the estimated required number of process units.

For the production of each type of parts, the appropriate equipment must be involved. The service system in our task will consist of $\mathrm{m}$ processors from the set $\left\{P_{1}, P_{2}, \ldots, P_{m}\right\}$, which correspond to each process operation. Jobs $T_{i}, i=\overline{1, n}$ come into the system at the moment of the time $d_{i}$, in case of simultaneous arrival, the moment of arrival time is fixed $d_{1}=d_{2}=\ldots=0$.

Maintenance of the Ti job includes $r_{i}$ consecutive stages, at stage $q, q=\overline{1, r_{i}}$, the $T_{i}$ job is serviced by the $P_{q}^{i}$ processor, $P_{q}^{i} \in\left\{P_{1}, P_{2}, \ldots, P_{m}\right\}, 1 \leq q \leq r_{i}$, are not necessarily different. The process of servicing a job by a separate processor at some particular stage is called an operation. In this terminology, the process of servicing the $T_{i}$ job consists in sequentially performing $r_{i}$ operations, i.e. passing the part (object) through the processor is a process operation.

For each $T_{i}$ job, $i=\overline{1, n}$, the processor paths are assumed to be set and may be different for different jobs.

The route for the job is described by the following expression:

$$
L^{i}=\left(P_{1}^{i}, P_{2}^{i}, \ldots, P_{q}^{i}, \ldots, P_{r_{i}}^{i}\right), q=\overline{1, r_{i}}
$$

Each processor serves jobs sequentially, with no more than one job at each point in time.

In the terminology of process operations, the maintenance process of the $T_{i}$ job consists in sequentially performing $r_{i}$ operations, each of which is characterized by an ordered triple of numbers $(i, P, q)$, where $i=\overline{1, n} ; P \in\left\{P_{1}, P_{2}, \ldots, P_{m}\right\} ; q=\overline{1, r_{i}}$.

Duration $t_{i p q}>0$ of execution of all process operations is assumed to be specified.

When the maintenance of the $T_{i}$ job is completed, the processor $P_{l}$ writes as:

$$
t_{i P q}^{\text {end }}=t_{i P q}^{\text {start }}+t_{i P q} \text {, }
$$

where $t_{i P q}^{\text {start }}$ - is the start time of the $T_{i}$ job service by processor $P_{l}$.

Consider servicing multiple jobs on systems with different routes without interruptions. If the process of servicing the job $T_{i}$ by the processor $P_{l}$ starts at a time $t_{i l}^{\text {start }}$, it proceeds continuously and ends at a time $t_{i l}^{\text {end }}=t_{i l}^{\text {start }}+t_{i l}$.

Each processor can simultaneously serve no more than one job, each job can simultaneously be served by no more than one processor.

Part manufacturing routines help you record the process flow for each part type. Adhering to this plan, for each part we obtain the technological process of its passage of processors (machines) corresponding to a certain technological operation, that is, the flow routes of processors during the processing of parts.

Part 491.SMK.

1. A hoop place.

2. Lathe.

3. Mandrel.

4. Workplace.

5. Mandrel.

6. Workplace.

7. Mandrel.

8. Workplace.

9. Balancing.

10. Painting.

11. Workplace. 
Part disk.

1. Lathe.

2. Workplace.

3. Layout plate.

4. Mandrel.

5. Workplace.

6 . The stand.

Part cover.

1. Lathe.

2. Workplace.

3. Mandrel.

4. Workplace.

5. Layout plate.

6. Mandrel.

7. Workplace.

8. The stand.

Thus, a 8 of machines is involved in the process, that is, the process is provided by a set of processors $P=\left\{P_{1}, P_{2}, \ldots, P_{8}\right\}$, which are different in terms of capabilities, speed and functional operations. Processor route for parts of the first type, i.e. for jobs from the set $T^{1}$ is as follows:

$$
\begin{aligned}
L^{1}= & \left(P_{1}^{1}, P_{2}^{1}, P_{3}^{1}, P_{4}^{1}, P_{5}^{1}, P_{6}^{1}, P_{7}^{1}, P_{8}^{1}, P_{9}^{1}, P_{10}^{1}, P_{11}^{1}\right)= \\
& =\left(P_{1}, P_{2}, P_{3}, P_{4}, P_{3}, P_{4}, P_{3}, P_{4}, P_{5}, P_{6}, P_{4}\right)
\end{aligned}
$$

for parts of the second type, that is, for jobs of the set $T^{2}$ :

$$
L^{2}=\left(P_{2}, P_{4}, P_{7}, P_{3}, P_{4}, P_{8}\right)
$$

for third-type parts, i.e. for jobs from the set $T^{3}$ :

$$
L^{3}=\left(P_{2}, P_{4}, P_{3}, P_{4}, P_{7}, P_{3}, P_{4}, P_{8}\right)
$$

Adhering to the entered designations, we can say that $P_{q}^{i}$ with $q=9, i=1$ will be $P_{9}^{1}=P_{5}$, similarly, you can define each processor number from the processor path for each part. The lengths of passage by the processor parts are known, which means that the moments of completion of servicing the part on the processor at a certain stage of the process are known.

The schedule of operation of the service system will be a set of indications as to which jobs were, are or will be serviced by which processors at each point in time. Our task is to consider all permissible schedules, that is, those that meet all the limitations arising from setting the task. Let schedule $\mathrm{S}$ correspond to the vector of times of completion of maintenance of jobs at this schedule

$$
\bar{t}(s)=\left(t_{1}^{\text {end }}, t_{2}^{\text {end }}, \ldots, t_{n}^{\text {end }}\right)
$$

Suppose that

$$
t_{1}^{\text {end }}=x_{1}, t_{2}^{\text {end }}=x_{2}, \ldots, t_{n}^{\text {end }}=x_{n}
$$

then $\bar{t}(s)=\left(x_{1}, x_{2}, \ldots, x_{n}\right)$.

Let's build a real function of $n$ variables

$$
F(x)=F\left(x_{1}, x_{2}, \ldots, x_{n}\right)=\left(t_{1}^{\text {end }}(s), t_{2}^{\text {end }}(s), \ldots, t_{n}^{\text {end }}(s)\right)
$$


Of all valid schedules, you must find the optimal schedule - the one that corresponds to the lowest value of $F(x): F(x) \rightarrow \min$ under the restrictions imposed on the times of completion of jobs $t_{i P q}^{\text {end }}$ :

for jobs from the set $T^{1}$

$$
t_{1 P_{1} 1}^{\text {end }} \leq t_{1 P_{2} 2}^{\text {end }} \leq t_{1 P_{3} 3}^{\text {end }} \leq t_{1 P_{4} 4}^{\text {end }} \leq t_{1 P_{3} 5}^{\text {end }} \leq t_{1 P_{4} 6}^{\text {end }} \leq t_{1 P_{3} 7}^{\text {end }} \leq t_{1 P_{4} 8}^{\text {end }} \leq t_{1 P_{5} 9}^{\text {end }} \leq t_{1 P_{6} 10}^{\text {end }} \leq t_{1 P_{4} 11}^{\text {end }}
$$

for jobs from the set $T^{2}$

$$
t_{2 P_{2} 1}^{\text {end }} \leq t_{2 P_{4} 2}^{\text {end }} \leq t_{2 P_{7} 3}^{\text {end }} \leq t_{2 P_{3} 4}^{\text {end }} \leq t_{2 P_{4} 5}^{\text {end }} \leq t_{2 P_{8} 6}^{\text {end }}
$$

for jobs from the set $T^{3}$

$$
t_{3 P_{2} 1}^{\text {end }} \leq t_{3 P_{4} 2}^{\text {end }} \leq t_{3 P_{3} 3}^{\text {end }} \leq t_{3 P_{4} 4}^{\text {end }} \leq t_{3 P_{7} 5}^{\text {end }} \leq t_{3 P_{3} 6}^{\text {end }} \leq t_{3 P_{4} 7}^{\text {end }} \leq t_{3 P_{8} 8}^{\text {end }}
$$

In order to minimize the total service time of all jobs in the system, it is necessary to find the lowest value of function $\overline{t_{\max }}(s)$, that is, show at which schedule the latest time of completion of service of any job is minimal.

\section{Conclusions}

A minimum-length schedule allows you not only to complete all jobs as early as possible, but also ensures maximum use of system resources. Minimizing schedule length minimizes downtime, which in turn leads to maximum utilization of processors.

This article provides a model of a certain process, which, despite its simplicity, is so meaningful that it encompasses a wide range of practical streamlining tasks that go beyond the initial wording.

\section{References}

Dudek, R.A., Smith, M.L., Panwalkar, S.S. (1974). Use of a case study in sequencing/ scheduling research. Baltimore: Johns Hopkins University, OMEGA.

Hefetz N., Adiri I. (1982). An efficient optimal algorithm for the two-machines, unit-time, jobshop, schedule-length, problem. Math. Oper. Res.

Mikhalevich, V.S., Kuksa A.I. (1983). Metodi posledovatelnoy optimizacii v diskretnikh setevikh zadachakh optimalnogo raspredeleniya resursov [Sequential optimization methods in discrete network tasks of optimal resource allocation]. Moskva: Nauka. [in Russian].

Van Dam J.P., Gaalman G.J.C., Sierksma G. (1994) Possibilities and Limitations of Using Scheduling Theory in the Process Industry. Springer, Berlin, Heidelberg: Operations Research Proceedings (Gesellschaftfür Operations Research e.V.), vol 1993.

Fisher M.L., Lageweg B.J., Lenstra J.K. (1983) Surrogate duality relaxation for job shop scheduling. Elsevier, Amsterdam: Discrete Applied Mathematics 